\title{
Estudio del Léxico Especializado en Inglés como Lengua Extranjera en Estudiantes de Pregrado
}

\author{
Angie E. Quintanilla(1,2)* y Pedro A. Salcedo(3) \\ (1) Facultad de Ciencias de la Educación, Universidad San Sebastián, Concepción, Chile. \\ (e-mail: angie.quintanilla@uss.cl) \\ (2) Programa de Doctorado en Lingüística, Universidad de Concepción, Chile. \\ (3) Departamento de Metodología de Investigación e Informática Educacional, Facultad de Educación Universidad de \\ Concepción, Concepción, Chile. (e-mail: psalcedo@udec.cl)
}

${ }^{*}$ Autor a quien debe ser dirigida la correspondencia

Recibido Nov. 6, 2018; Aceptado Ene. 16, 2019; Versión final Mar. 20, 2019, Publicado Ago. 2019

\begin{abstract}
Resumen
Se presentan los resultados de un estudio de disponibilidad léxica en el cual se encuestó a 350 estudiantes de pedagogía en inglés ( $1^{\circ}-4^{\circ}$ año) en 4 centros de interés de tipo especializado: planificación de clases, métodos y enfoques de enseñanza de inglés, evaluación y reflectividad. A pesar de la riqueza de los estudios existentes en el área de disponibilidad léxica, el léxico de las comunidades científicas y/o profesionales ha pasado casi inadvertido para los investigadores. El objetivo de esta investigación es conocer el léxico especializado de estos estudiantes en distintas etapas de su formación inicial docente. Los resultados evidencian que a medida que los estudiantes avanzan en sus estudios el léxico aumenta y se hace más cohesionado. Esto aumenta su nivel de comunicación, lo que se evidencia a través de un incremento sostenido del índice de disponibilidad léxica individual. Este aumento podría explicarse a partir de los conocimientos adquiridos y las experiencias de aprendizaje vividas durante su proceso de formación inicial docente.
\end{abstract}

\section{Study of the Specialized Lexicon in English as a Foreign Language in Undergraduate Students}

\begin{abstract}
This paper presents the results of a lexical availability study in which 350 English pedagogy students $\left(1^{\text {st }}-4^{\text {th }}\right.$ year) were surveyed in 4 specialized interest centers: lesson planning, methods and approaches to English teaching, assessment and reflectivity. Despite the wealth of existing studies in the area of lexical availability, the lexicon of the scientific and/or professional communities has gone almost unnoticed by researchers. The objective of this research is to know the specialized lexicon of these students in different stages of their initial teacher training. The results show that as the students advance in their studies, the lexicon increases and becomes more cohesive. This increases their level of communication, which evidences through a sustained increase in the individual lexical availability index. This increase could be explained by the knowledge acquired and the learning experiences lived during their teacher training process.
\end{abstract}




\section{INTRODUCCIÓN}

La disponibilidad léxica (DL) se inserta en el campo de la léxico-estadística, el cual se centra en el estudio cuantitativo del léxico mediante el análisis de frecuencia de palabras. La aplicación de este tipo de técnicas permite conocer el léxico de una comunidad de hablantes determinada. Los estudios en el área de la disponibilidad léxica se han centrado principalmente en conocer el léxico común utilizado por diferentes comunidades lingüísticas. Destaca en este contexto el proyecto panhispánico cuyo objetivo es la publicación del léxico disponible común de aquellos países que forman parte del mundo hispano (Ferreira et al., 2014). Las áreas temáticas o centros de interés en los cuales se ha enfocado el estudio de la disponibilidad léxica en español son 16: partes del cuerpo, la ropa, partes de la casa, los muebles de la casa, alimentos y bebidas, objetos colocados en la mesa para la comida, la cocina y sus utensilios, la escuela: muebles y materiales, iluminación, calefacción y medios de airear un recinto, la ciudad, el campo, medios de transporte, trabajos del campo y del jardín, los animales, juegos y distracciones y profesiones y oficios (Hernandez et al., 2006). Cabe señalar que algunos autores han agregado otros centros de acuerdo a sus intereses particulares, por ejemplo, sentimientos (Valencia y Echeverría, 1999), la inteligencia (Hernández, 2004), los colores (Valencia, 2010), terrorismo-crimen y salud-medicina (Ferreira y Echeverría, 2014), emociones (Jiménez y Dewaele, 2017), nuevas tecnologías: tic y educación (Herranz, 2018), entre otros.

La investigación en el área disponibilidad léxica "se ha dedicado a extraer el léxico de uso que se considera general, es decir, el léxico que cualquier tipo de hablante necesita para desenvolverse sin problemas dentro de una comunidad lingüística" (Urzúa et al., 2006). Sin embargo, el estudio del léxico en comunidades profesionales ha sido escaso. Al respecto, Urzúa at al. (2006) y Navarro (2009) señalan la necesidad de conocer qué ocurre con el léxico disponible de hablantes que se desenvuelven contextos especializados y que son parte de una comunidad de habla que utiliza un léxico técnico y/o científico para interactuar de forma efectiva con otros miembros al interior de dicha comunidad. En el proceso de formación inicial docente de estudiantes de pedagogía en inglés la adquisición de conocimiento pedagógico y disciplinar, y por consiguiente léxico, se sustenta en la Instrucción Basada en Contenidos, en la cual el estudiante adquiere de forma simultánea el conocimiento de disciplinas no lingüísticas y las habilidades comunicativas de la lengua meta, en este caso el inglés. Al respecto, Stoller (2007) señala que "el aprendizaje del contenido ayuda al aprendizaje de la lengua, mientras que el dominio de la lengua facilita al estudiante el acceso al contenido en su formación profesional o en su área ocupacional" (p. 59). Por otro lado, autores como Bonorino y Cuñarro (2006) sostienen que los procesos de aprendizaje se sustentan en la adquisición del léxico, y que sería esta adquisición de léxico "especializado" la que permitiría a los futuros docentes acceder a los conocimientos propios de la disciplina. En esta misma línea, Valencia et al. (1992) afirman que el léxico es parte esencial del conocimiento lingüístico y su "manejo instrumental pleno" resulta fundamental en procesos de aprendizaje. Finalmente, Herranz (2018) manifiesta que "el vocabulario es un aspecto vital para la enseñanza-aprendizaje de cualquier concepto o materia". Por esta razón, se realiza el análisis de la disponibilidad léxica de 350 estudiantes de pedagogía en inglés con el propósito de conocer su léxico especializado en distintas etapas de su formación académica.

\section{Disponibilidad léxica}

Los estudios en el área de la disponibilidad léxica comienzan en Francia en los años cincuenta con el objetivo de elaborar una "lengua base", en términos léxicos y gramaticales, para ser enseñada a inmigrantes y a habitantes de excolonias francesas.

Con respecto al vocabulario necesario para esta "lengua base", este proyecto se centró en el léxico disponible, el cual de acuerdo a Gougenheim (1967), corresponde a aquellas palabras que forman parte del lexicón mental de un hablante y que se utilizan solo cuando las circunstancias comunicativas lo requieren. En este mismo contexto, Michéa (1953) indica que una palabra disponible puede no ser frecuente, sin embargo, se presentaría en la mente del hablante rápidamente en el momento que fuera necesario utilizarla.

El léxico disponible se obtiene a partir de pruebas de asociación en las cuales se presenta un centro de interés (estímulo) que evocaría el léxico potencial del hablante. A partir de la presentación de este centro de interés, se les solicita a los sujetos enunciar o escribir palabras asociadas con la temática que se les ha presentado en un tiempo de 2 minutos $(2,1986)$. Una de las fórmulas más utilizadas para determinar la disponibilidad léxica de una población es la propuesta por Lorán y López (1983):

$$
D(p)=\frac{f_{1}+\lambda f_{2}+\lambda^{2} f_{3}+\ldots+\lambda^{n-1} f_{n}}{N}
$$

Dónde: $\mathrm{D}(\mathrm{p})$ : Índice de disponibilidad léxica de la palabra p, N: Número de sujetos encuestados, Fi: Frecuencia de la palabra en la posición $\mathrm{i}$, con $1<\mathrm{i}<\mathrm{n}$ y $\lambda$ : Factor de ponderación (con un valor de 0,90 ). Esta fórmula 
permite establecer un índice de disponibilidad léxica (IDL) para cada palabra entregada por la población en estudio. Este índice sirve como un indicador que determina cuán disponible está cada vocablo en la mente de los hablantes. A partir de este dato es posible generar listados de palabras ordenadas de mayor a menor de acuerdo a su IDL, las primeras palabras de esta lista corresponderían a las primeras palabras que vendrían a la mente de los hablantes cuando se enfrentan a una temática específica (centro de interés).

\section{Disponibilidad léxica especializada}

De acuerdo a López (1999), los vocablos que posee un hablante se actualizan cuando es necesario comunicar información específica. Por esta razón, autores como Urzúa et al. (2006) y Navarro (2009) creen que es necesario estudiar el léxico de comunidades que utilicen léxico técnico y/o científico como base para su interacción. En el caso de estas comunidades, el léxico disponible es restrictivo cuantitativamente y cualitativamente dado que se limita a una comunidad de habla especifica dentro de una comunidad de habla mayor (Navarro, 2009).

En el área del léxico especializado los estudios han sido escasos y se han focalizado en los medios de comunicación (Gómez y Guerra, 2004), en las matemáticas (Ferreira et al., 2014; Rojas et al., 2017; Salcedo et al., 2016; Salcedo y Del Valle, 2013; Salcedo et al., 2013; Urzúa et al., 2006), en el área jurídica (Medina, 2009) y en fisioterapia (Navarro, 2009). Desde el punto metodológico, estos estudios, en general, centran su interés en estudiar cómo evoluciona el léxico especializado y/o específico a medida que aumentan los años de estudio de los sujetos que forman parte de la muestra. Otra arista que se ha desarrollado en esta área consiste en contrastar el léxico de estudiantes con expertos, que pueden ser profesores y/o profesionales en ejercicio (Urzúa et al., 2006). Por otra parte, se ha investigado a estudiantes preuniversitarios de acuerdo al tipo de especialización académica recibida previo al ingreso a la universidad, con el propósito de determinar si existen diferencias en su léxico (Hernández, 2015). Es importante señalar que los estudios de disponibilidad léxica específica o especializada son aún escasos. Por lo tanto, el potencial de este tipo de investigaciones es desconocido y necesita ser profundizado dado las implicancias pedagógicas y metodológicas que podría tener en diversas áreas del saber.

\section{Índice de disponibilidad léxica individual (idli)}

La investigación en el área de disponibilidad léxica se han centrado, en general, en determinar el léxico de comunidades de hablantes, es decir, se ha optado por favorecer el estudio de un comportamiento social por sobre el individual. Sin embargo, también es posible utilizar la léxico-estadística con el propósito de inferir comportamientos individuales. Autores como López y Strassburger (1991) confeccionaron formas de cálculo de la disponibilidad léxica individual (IDLi) con el propósito de analizar la importancia de la producción léxica individual en la disponibilidad total de una muestra de hablantes. La hipótesis de estos investigadores en que si un sujeto actualiza los términos de mayor índice de disponibilidad léxica probablemente tendrá más disponibilidad léxica que otro. A continuación, se presenta la fórmula para el cálculo del IDLi desarrollada por López y Strassburger (1991) es la siguiente:

$$
D(S i)=\frac{1}{k} \sum_{j=1}^{n} d\left(P_{i j}\right) \cdot e^{-2.3\left\langle\frac{j-1}{n-1}\right\rangle}
$$

Dónde: $\mathrm{D}(\mathrm{Si})=$ disponibilidad del sujeto $\mathrm{i} ; \mathrm{d}(\mathrm{Pij})=$ disponibilidad de la palabra respondida por el sujeto $\mathrm{i}$ en la posición j; $n=$ máximo número de respuestas del centro de interés en cuestión; y $k=$ constante para ajustar las calificaciones. De acuerdo a Callealta y Gallego (2016), el índice de disponibilidad léxica individual (IDLi) permite medir "el grado de aportación de un individuo dentro de los listados generales de disponibilidad" (p.44) o "el grado de disponibilidad léxica que tiene un informante dentro de un grupo" (p.42). En otras palabras, a mayor IDLi de un sujeto mayor sería su aporte al léxico del grupo y, por consiguiente, mejores serían sus posibilidades de comunicarse con el mismo.

\section{METODOLOGÍA}

Esta investigación se enmarca en un diseño no-experimental ya que no se manipulan variables en estudio. En los estudios de disponibilidad léxica solo se provee un estímulo a través de una prueba asociativa controlada en contexto de laboratorio para activar la producción de elementos léxicos en los estudiantes. Trecientos cincuenta estudiantes de pregrado cursando la carrera de pedagogía en inglés formaron parte de esta investigación. La muestra se conforma de la siguiente manera: 118 de $1^{\circ}$ año, 93 de $2^{\circ}$ año, 78 de $3^{\circ}$ año y 61 de $4^{\circ}$ año. 
La recolección de datos se realizó a partir de la encuesta de disponibilidad léxica que se observa en la figura 1. Las instrucciones de la encuesta se leen en voz alta, y luego se presentan cada uno de los centros de interés, precedidos de la frase "Escribe todas las palabras relacionadas con .......... que tú conoces". Para cada centro de interés se asigna un tiempo de 2 minutos, de acuerdo a la tendencia actual en disponibilidad léxica (Garzón y Penagos, 2016).

Datos del informante:

1. Sexo: Hombre Mujer

2. Edad:

3. Año de ingreso al programa:

Instrucciones:

Tendrás 2 minutos para escribir todas las palabras en inglés que se te vengan a la mente en cada una de las 4 temáticas que presentará el encuestador.

No existe un mínimo ni un máximo de palabras esperadas, sólo debes escribir las palabras que se activen en tu memoria. Ejemplo:

Encuestador: Write all the words related to "Transport" you know.

\begin{tabular}{|l|}
\hline \multicolumn{1}{|c|}{ Transport } \\
\hline 1. Car \\
\hline 2. Bus \\
\hline 3. Train \\
\hline (......) \\
\hline 25. Airplane \\
\hline
\end{tabular}

\begin{tabular}{|l|l|l|l|}
\hline & & & \\
\hline 1. & 1. & 1. & 1. \\
\hline 2. & 2. & 2. & 2. \\
\hline 3. & 3. & 3. & 3. \\
\hline$(\ldots .)$. & $(\ldots \ldots)$ & $(\ldots)$. & $(\ldots)$. \\
\hline 25. & 25. & 25. & 25. \\
\hline
\end{tabular}

Fig. 1: Encuesta de disponibilidad léxica.

Los centros de interés encuestados fueron los siguientes: planificación de clases, métodos y enfoques de enseñanza de inglés, evaluación y reflectividad. Estos centros se definen como sigue:

Planificación de clases: proceso de especificación de la secuencia de actividades prevista para un período temporal limitado, mediante las cuales se pretende conseguir los objetivos de enseñanza fijados en la planificación global para un curso. Enfoques y métodos en la enseñanza de lenguas: conjunto de procedimientos basados en diferentes puntos de vista sobre qué es la lengua y cómo se aprende. Evaluación: recolección sistemática de datos empíricos sobre el aprendizaje de los estudiantes con el propósito de mejorar los aprendizajes. Reflectividad: parte de un proceso que consiste en mirar lo que se hace en el aula, pensar en las razones por las que esto se hace, y ver el efecto de estas decisiones. Consiste en un proceso de autoobservación y auto-evaluación.

El procesamiento de los datos obtenidos se realizó utilizando el programa Dispogen II (Echeverría et al., 2005) con el propósito de calcular índices y frecuencias, considerando los tres estadígrafos que permiten establecer con mayor claridad la riqueza léxica de los sujetos que conforman la muestra (Urzúa et al., 2006): número de vocablos (NPD), el promedio de palabras (XR) y el índice de cohesión (IC). Ferreira et al. (2014) describen estos índices como:

NPD: El número de vocablos o palabras diferentes conocidos por el grupo muestral; XR: El número de vocablos que, en promedio, poseen los sujetos para su comunicación en el ámbito de conocimiento que explora el centro de interés respectivo; IC: El indicador del grado de coincidencia en las respuestas de los sujetos. Este índice es el resultado del cuociente entre el promedio de vocablos y el número de vocablos diferentes. La fórmula del índice de cohesión es la siguiente: IC = XR/NPD (Echeverría et al., 1987). Además de estos tres índices, se consideró el índice de disponibilidad léxica individual (IDLi), el cual permite medir el aporte que hace cada individuo al léxico del grupo. 


\section{RESULTADOS}

A continuación, se presentan los resultados obtenidos a partir del análisis de disponibilidad léxica de los sujetos de la muestra.

La Tabla 1 presenta los resultados generales obtenidos en los tres índices NPD, XR e IC de cada centro y en los cuatro niveles en estudio. A partir de estos datos es posible observar que el NPD no se comporta del modo esperado, es decir, no se evidencia que a mayor cantidad de años de estudio exista mayor producción de vocablos.

Con respecto al XR y al IC es posible señalar que en general, estos valores crecen a medida que aumenta el nivel de los estudiantes. Solo en el caso del centro de interés "Enfoques y métodos en la enseñanza de lenguas" se observa un XR de palabras más alto en estudiantes de segundo año $(X R=6,59)$ que de tercer año $(X R=5,37)$. Esto podría explicarse, de acuerdo a Luque (2004), debido a los constantes cambios a los cuales está sujeto el léxico que posee el individuo, los cuales se relacionan con el entorno y con las nuevas experiencias a las cuales se enfrenta. En este caso en particular, los estudiantes de segundo año cursan una asignatura que tiene como eje central los enfoques y métodos de la enseñanza de inglés. Por otro lado, el IC refleja que en los primeros años de estudios existe un mayor grado de dispersión o un menor grado de coincidencia en los vocablos aportados por la muestra, lo que es provocado por el alto NPD. Sin embargo, en cuarto año se evidencia un mayor IC que evidenciaría un mayor grado de homogenización en el léxico de los estudiantes, especialmente en el área de planificación de clases y enfoques y métodos.

Tabla 1: Índices generales en los distintos niveles en estudio.

\begin{tabular}{|c|c|c|c|c|c|}
\hline Índice & Nivel & Plan & Metod & Eval & Refl \\
\hline \multirow{4}{*}{ NPD } & Primer año & 261 & 164 & 223 & 208 \\
\cline { 2 - 6 } & Segundo año & 337 & 142 & 308 & 243 \\
\cline { 2 - 6 } & Tercer año & 299 & 118 & 304 & 272 \\
\cline { 2 - 6 } & Cuarto año & 235 & 118 & 278 & 228 \\
\hline \multirow{5}{*}{ XR } & Primer año & 6,55 & 3,97 & 6,00 & 4,04 \\
\cline { 2 - 6 } & Segundo año & 12,45 & 6,59 & 8,70 & 5,79 \\
\cline { 2 - 6 } & Tercer año & 13,87 & 5,37 & 9,29 & 7,20 \\
\cline { 2 - 6 } & Cuarto año & 14,98 & 7,37 & 11,86 & 7,52 \\
\hline \multirow{4}{*}{ IC } & Primer año & 0,02509 & 0,02423 & 0,02694 & 0,01943 \\
\cline { 2 - 6 } & Segundo año & 0,03694 & 0,04641 & 0,02827 & 0,02385 \\
\cline { 2 - 6 } & Tercer año & 0,04639 & 0,04552 & 0,03057 & 0,02648 \\
\cline { 2 - 6 } & Cuarto año & 0,06376 & 0,06251 & 0,04269 & 0,03300 \\
\hline
\end{tabular}

Con el objetivo de describir el léxico disponible de los sujetos de la muestra se presentan en orden decreciente, de acuerdo a IDL, los 15 vocablos más disponibles en los centros de interés estudiados en cada uno de los niveles examinados. La Tabla 2 presenta los vocablos del centro de interés "planificación de clases". En este primer centro se observa que en el caso de primer año las palabras disponibles son variadas y no están necesariamente relacionadas con la planificación de una clase, entre ellas encontramos actividad (activity), objetivo (objective) y otras relacionadas a material utilizado en clases (ppt, material, book, worksheet). Además, es posible observar la aparición del vocablo planification entre las 3 palabras más disponible que correspondería en inglés a una planificación de tipo económica y/o política, y no a una planificación de clase como sería el vocablo planning o lesson-planning. En el caso de los estudiantes de $2^{\circ}$ a $4^{\circ}$ año se evidencia léxico más especializado relacionado con los objetivos de la planificación (aim, maiaim, subsidiary-aim, personal-aim), etapas de una clase (engage, warm-up, development, presentation, practice, production, assessment) y conocimiento previo de los estudiantes (anticipated-problem, assumedknowledge), entre otras temáticas.

La Tabla 3 presenta los vocablos del centro de interés "enfoque y métodos en la enseñanza de lenguas". Al igual que en el centro anterior, los estudiantes de primer año presentan un léxico muy general, haciendo alusión a las 4 habilidades (speaking, listening, writing y reading) y a terminología general en el área de métodos y enfoques (methodology, method, approach). Por otro lado, Los estudiantes de $2^{\circ}$ a $4^{\circ}$ año presentan vocablos relacionados con métodos (TBL, audiolingualism, silent-way, gramar-translation, suggestopedia, direct-method, army-method y CLT), modelos de enseñanza (ESA y PPP), técnicas (TPR y drilling) y enfoques (natural-approach, communicative-approach, lexical-approach, dialogic-pedagogy, constructivism y behaviorism), entre otros. 
Tabla 2: Vocablos más disponibles en centro de interés "Planificación de clases".

\begin{tabular}{|l|l|l|l|}
\hline \multicolumn{1}{|c|}{ Primero } & \multicolumn{2}{c|}{ Tegundo } & \multicolumn{1}{c|}{ Cuarto } \\
\hline Activity & Aim & Objective & Subsidiary-Aim \\
\hline Time & Time & Time & Aim \\
\hline Planification & Activity & Aim & Time \\
\hline Objective & Objective & Activity & Main-Aim \\
\hline Content & Student & Material & Objective \\
\hline Material & Teacher & Warm-Up & Anticipated-Problem \\
\hline Planning & Material & Student & Assessment \\
\hline PPT & PPP & Main-Aim & Student \\
\hline Student & Practice & Timing & Aid \\
\hline Writing & Engage & Subsidiary-Aim & Activity \\
\hline Book & Content & Approach & Personal-Aim \\
\hline Worksheet & Presentation & PPP & Assumed-Knowledge \\
\hline Schedule & Method & Teacher & Development \\
\hline Homework & Approach & Content & Teacher \\
\hline Listening & Production & Engage & Timing \\
\hline
\end{tabular}

Tabla 3: Vocablos más disponibles en centro de interés "Enfoques y métodos en la enseñanza de lenguas".

\begin{tabular}{|l|l|l|l|}
\hline \multicolumn{1}{|c|}{ Primero } & \multicolumn{1}{|c|}{ Segundo } & \multicolumn{1}{c|}{ Tercero } & \multicolumn{1}{c|}{ Cuarto } \\
\hline Speaking & Suggestopedia & TPR & TBL \\
\hline Activity & Audiolingualism & PPP & PPP \\
\hline Listening & PPP & Audiolingualism & TPR \\
\hline Writing & TPR & TBL & Audiolingualism \\
\hline Method & Silent-Way & Suggestopedia & $\begin{array}{l}\text { Communicative- } \\
\text { Approach }\end{array}$ \\
\hline Teaching & Grammar-Translation & $\begin{array}{l}\text { Communicative- } \\
\text { Approach }\end{array}$ & Silent-Way \\
\hline Reading & Natural-Approach & Silent-Way & Grammar-Translation \\
\hline Approach & TBL & Grammar-Translation & ESA \\
\hline Learning & CLT & Lexical-Approach & Suggestopedia \\
\hline Grammar & Direct-Method & Natural-Approach & CLT \\
\hline Planning & $\begin{array}{l}\text { Communicative- } \\
\text { Approach }\end{array}$ & Direct-Method & Lexical-Approach \\
\hline Methodology & Lexical-Approach & CLT & Dialogic-Pedagogy \\
\hline Material & TTT & Dialogic-Pedagogy & Constructivism \\
\hline Group-Work & Army-Method & CAE & Behaviorism \\
\hline Communication & ESA & Eclectic-Approach & Drilling \\
\hline
\end{tabular}

La Tabla 4 presenta los vocablos del centro de interés "evaluación". Nuevamente, en el caso de los estudiantes de primer año, los vocablos más disponibles se relacionan con instrumentos o instancias de evaluación (test, quiz, exam, rubric, oral-test, oral-presentation), habilidades a evaluar (writing, speaking y listening) y elementos varios de una evaluación como, por ejemplo; mark o grade (calificación obtenida) y point (puntaje obtenido). Un fenómeno bastante parecido se observa en $2^{\circ}$ y $3^{\circ}$ año donde nuevamente aparecen entre los vocablos más disponibles, instancias de evaluación, elementos varios de una evaluación (score=puntaje), se agregan ítems de evaluación (multiple-choice y question), formato de evaluación (written y oral). Sin embargo, en cuarto año descatan vocablos relacionados con el tipo de evaluación (formalassessment e informal-assessment) y función de la evaluación (summative-test, formative-test). Cabe destacar que en todos los niveles aparece el vocablo feedback (retroalimentación), lo cual deja de manifiesto que los sujetos de la muestra comprenden de una u otra manera la importancia de este elemento en los procesos evaluativos. 
Tabla 4: Vocablos más disponibles en centro de interés "Evaluación".

\begin{tabular}{|l|l|l|l|}
\hline \multicolumn{1}{|c|}{ Primero } & \multicolumn{2}{c|}{ Tegundo } & \multicolumn{1}{c|}{ Cuarto } \\
\hline Test & Test & Test & Formal-Assessment \\
\hline Mark & Mark & Evaluation & Informal-Assessment \\
\hline Evaluation & Evaluation & Feedback & Test \\
\hline Quiz & Grade & Rubric & Rubric \\
\hline Exam & Rubric & Mark & Mark \\
\hline Point & Score & Grade & Formative \\
\hline Grade & Teacher & Oral & Feedback \\
\hline Rubric & Student & Quiz & Summative-Test \\
\hline Writing & Exam & Written & Informal \\
\hline Feedback & Feedback & Formative & Formal \\
\hline Oral & Oral-Test & Homework & Summative \\
\hline Oral-Test & Multiple-Choice & Evaluate & Student \\
\hline Oral-Presentation & Homework & Writing & Formative-Test \\
\hline Speaking & Question & Oral-Presentation & Evaluation \\
\hline Listening & Formative & Help & Monitoring \\
\hline
\end{tabular}

La Tabla 5 presenta los vocablos del centro de interés "reflectividad". Los estudiantes de primer año entregan vocablos generales, como paz (peace), yoga, mente (mind) e incluyen algunos más como son: pensamiento (thinking) y reflexión (reflection). En el caso de los estudiantes de $2^{\circ}$ año, se incluye la idea de la problemática (problem y mistake), la mejora (improvement y feedback) y la experiencia (experience y belief). En el caso de los estudiantes de $3^{\circ}$ y $4^{\circ}$ año, se incluyen vocablos relacionados desarrollo de pensamiento (thinking, criticalthinking y analysis) y con prácticas reflexivas de autocrítica (self-evaluation, self-reflection, self-assessment, feedback y reflecting).

Tabla 5: Vocablos más disponibles en centro de interés "Reflectividad".

\begin{tabular}{|l|l|l|l|}
\hline \multicolumn{1}{|c|}{ Primero } & \multicolumn{1}{c|}{ Tercero } & \multicolumn{1}{c|}{ Cuarto } \\
\hline Thinking & Thinking & Thinking & Thinking \\
\hline Reflection & Portfolio & Thought & Reflection \\
\hline Mirror & Reflection & Mirror & Self-Evaluation \\
\hline Thought & Thought & Reflection & Feedback \\
\hline Perception & Improvement & Improvement & Reflecting \\
\hline Feeling & Idea & Feedback & Improvement \\
\hline Mistake & Mirror & Learning & Self-Assessment \\
\hline Feedback & Feedback & Critical-Thinking & Thought \\
\hline Mind & Problem & Analysis & Opinion \\
\hline Peace & Mistake & Brain & Solution \\
\hline Primero & Segundo & Tercero & Cuarto \\
\hline Yoga & Experience & Writing & Personal \\
\hline Yourself & Class & Self-Evaluation & Self-Reflection \\
\hline Myself & Belief & Analyzing & Aim \\
\hline Learning & Opinion & Conclusion & Conclusion \\
\hline Writing & Reflecting & Portfolio & Development \\
\hline
\end{tabular}

Con respecto al índice de disponibilidad léxica individual (IDLi), la tabla 6 muestra las medias del IDLi para cada nivel y centro, encontrando un aumento sostenido al aumentar el nivel. Sin embargo, para probar que este aumento no es debido al azar se debe utilizar una prueba estadística como ANOVA, la cual tiene como requisito que exista normalidad en la variable. Al estudiar la normalidad del IDLi por centro y grado (tabla 7), 
nos encontramos que al aplicar la prueba de Kolmogorov, no es posible aceptar la normalidad para todos los niveles y centros en estudio, es decir, no se cumple en todos los casos que $p$ (Sig.) $>0,05$, lo que nos lleva a buscar una prueba alternativa a ANOVA para realizar el contrastar la hipótesis, de diferencia entre las medias.

Tabla 6: Promedio de IDLi por cada centro

\begin{tabular}{|c|c|c|c|c|}
\hline Nivel & $\begin{array}{c}\text { Promedio del IDLi } \\
\text { Planificacion }\end{array}$ & $\begin{array}{c}\text { Promedio del IDLi } \\
\text { Métodos }\end{array}$ & $\begin{array}{c}\text { Promedio del IDLi } \\
\text { Evaluación }\end{array}$ & $\begin{array}{c}\text { Promedio del IDLi } \\
\text { Reflectividad }\end{array}$ \\
\hline Primero &, 3166 &, 1815 &, 4732 &, 4400 \\
\hline Segundo &, 7357 &, 7403 &, 4290 &, 2913 \\
\hline Tercero &, 8441 &, 8089 &, 4243 &, 4419 \\
\hline Cuarto & 1,1164 & 1,4488 &, 8368 &, 3736 \\
\hline
\end{tabular}

Tabla 7: Prueba de normalidad

\begin{tabular}{|c|c|c|c|c|}
\hline & \multirow{2}{*}{ Nivel } & \multicolumn{3}{|c|}{ Kolmogorov-Smirnova } \\
\hline & & Estadístico & $g l$ & Sig. \\
\hline \multirow{4}{*}{$\begin{array}{l}\text { Media_IDLi } \\
\text { Planificación }\end{array}$} & Primero & ,095 & 118 &, 011 \\
\hline & Segundo &, 055 & 93 &, $200^{*}$ \\
\hline & Tercero &, 058 & 78 & ,200* \\
\hline & Cuarto & ,057 & 61 &, $200^{*}$ \\
\hline \multirow{4}{*}{$\begin{array}{l}\text { Media_IDLi } \\
\text { Métodos y enfoques }\end{array}$} & Primero &, 112 & 118 &, 001 \\
\hline & Segundo & ,103 & 93 &, 016 \\
\hline & Tercero & , 102 & 78 &, 043 \\
\hline & Cuarto & ,147 & 61 &, 002 \\
\hline \multirow{4}{*}{$\begin{array}{l}\text { Media_IDLi } \\
\text { Evaluación }\end{array}$} & Primero &, 084 & 118 &, 039 \\
\hline & Segundo & ,099 & 93 & ,024 \\
\hline & Tercero &, 083 & 78 & ,200* \\
\hline & Cuarto &, 063 & 61 & ,200* \\
\hline \multirow{4}{*}{$\begin{array}{l}\text { Media_IDLi } \\
\text { Reflectividad }\end{array}$} & Primero & ,217 & 118 &, 000 \\
\hline & Segundo & ,077 & 93 & ,200* \\
\hline & Tercero &, 092 & 78 & ,166 \\
\hline & Cuarto &, 057 & 61 & ,200* \\
\hline
\end{tabular}

Por lo anterior, para contrastar la hipótesis se ha seleccionado la prueba de Kruskal Wallis (tabla 8) la que nos permite finalmente aceptar la diferencia de medias entre los niveles para cada uno de los centros. Es decir, hemos probado que en la medida que aumenta el nivel, en cada uno de los centros, aumenta de la misma forma el IDLi, es decir al ser $p<0,05$ en cada caso, se rechaza la hipótesis de igualdad de medias. Por otro lado, si se hubiera utilizado ANOVA, suponiendo normalidad, el resultado habría sido el mismo (ver tabla 9). Se habría rechazado la hipótesis de igualdad de medias, al ser $p<0,05$ en cada caso. Como conclusión respecto al IDLi en cada centro, podemos decir que existe evidencia suficiente, para establecer que en la medida que aumenta de curso un alumno, este va adquiriendo mayor léxico del grupo y lo que le permite comunicarse de una forma más efectiva con la comunidad de hablantes en la cual se desenvuelve.

Tabla 8: Kruskal Wallis a) Prueba de Kruskal Wallis y b) Variable de agrupación: Nivel

\begin{tabular}{|l|r|r|r|r|}
\cline { 2 - 5 } \multicolumn{1}{c|}{} & $\begin{array}{c}\text { Media_IDLi } \\
\text { Planificación }\end{array}$ & $\begin{array}{c}\text { Media_IDLi } \\
\text { Métodos }\end{array}$ & $\begin{array}{c}\text { Media_IDLi } \\
\text { Evaluación }\end{array}$ & $\begin{array}{c}\text { Media_IDLi } \\
\text { Reflectividad }\end{array}$ \\
\hline Chi-cuadrado & 214,662 & 212,501 & 60,139 & 28,304 \\
gl & 3 & 3 & 3 & 3 \\
Sig. asintótica &, 000 &, 000 &, 000 &, 000 \\
\hline
\end{tabular}


Tabla 9: ANOVA

\begin{tabular}{|c|c|c|c|c|c|c|}
\hline & & $\begin{array}{l}\text { Suma de } \\
\text { cuadrados }\end{array}$ & $g l$ & $\begin{array}{c}\text { Media } \\
\text { cuadrática }\end{array}$ & $F$ & Sig. \\
\hline \multirow{3}{*}{$\begin{array}{l}\text { Media_IDLi } \\
\text { Planificación }\end{array}$} & Entre grupos & 29,581 & 3 & \multirow[t]{3}{*}{9,860} & \multirow[t]{3}{*}{171,452} & \multirow[t]{3}{*}{,000 } \\
\hline & $\begin{array}{l}\text { Dentro de } \\
\text { grupos }\end{array}$ & 19,899 & 346 & & & \\
\hline & Total & 49,479 & 349 & & & \\
\hline \multirow{3}{*}{$\begin{array}{l}\text { Media_IDLi } \\
\text { Métodos }\end{array}$} & Entre grupos & 66,965 & 3 & \multirow{3}{*}{$\begin{array}{r}22,322 \\
, 114\end{array}$} & \multirow[t]{3}{*}{196,271} & \multirow[t]{3}{*}{, 000} \\
\hline & $\begin{array}{l}\text { Dentro de } \\
\text { grupos }\end{array}$ & 39,350 & 346 & & & \\
\hline & Total & 106,315 & 349 & & & \\
\hline \multirow{3}{*}{$\begin{array}{l}\text { Media_IDLi } \\
\text { Evaluación }\end{array}$} & Entre grupos & 7,852 & 3 & \multirow{3}{*}{$\begin{array}{r}2,617 \\
, 070\end{array}$} & \multirow[t]{3}{*}{37,309} & \multirow[t]{3}{*}{,000 } \\
\hline & $\begin{array}{l}\text { Dentro de } \\
\text { grupos }\end{array}$ & 24,272 & 346 & & & \\
\hline & Total & 32,124 & 349 & & & \\
\hline \multirow{3}{*}{$\begin{array}{l}\text { Media_IDLi } \\
\text { Reflectividad }\end{array}$} & Entre grupos & 1,427 & 3 & \multirow{3}{*}{$\begin{array}{l}, 476 \\
, 045\end{array}$} & \multirow[t]{3}{*}{10,539} & \multirow[t]{3}{*}{, 000} \\
\hline & $\begin{array}{l}\text { Dentro de } \\
\text { grupos }\end{array}$ & 15,616 & 346 & & & \\
\hline & Total & 17,043 & 349 & & & \\
\hline
\end{tabular}

\section{DISCUSIÓN}

Los resultados encontrados dan cuenta de un aumento en el léxico de los sujetos de la muestra y en el IDLi a medida que aumenta el nivel de estudios. Esta evidencia coincide con lo que se esperaba encontrar dado que resultados similares fueron obtenidos en investigaciones que estudiaban sujetos en distintas etapas de desarrollo académico (Urzua et al., 2006; Salcedo y Del Valle, 2013; Ferreira et al., 2014; Rojas et al., 2017).

A partir de los hallazgos de esta investigación es posible señalar que la técnica de la disponibilidad léxica probaría ser una herramienta apropiada para medir el conocimiento que el estudiante va adquiriendo en su proceso de formación. Al respecto, el contraste entre los vocablos encontrados en los diferentes niveles en estudio $\left(1^{\circ}-4^{\circ}\right)$ y el orden en el cual estos aparecen en las listas evidencian "una clara tendencia sobre la transformación del léxico disponible hacia una disponibilidad de vocablos más técnicos y propios de cada área, a medida que permanecen más tiempo los alumnos en la universidad" (Rojas et al., 2017, p.11). Esto evidenciaría cambios en el lexicón mental de los estudiantes, el cual de acuerdo a Del Valle et al. (2016), "aumenta, disminuye y cambia dinámicamente, siendo permeado por el contexto que rodea al individuo y el momento de vida en el cual se encuentra" (p.145). Por otro lado, resultados como este permitirían tomar decisiones en planificación a nivel léxico y curricular, didáctica de aula y diseño de materiales didácticos (Šifrar Kalan, 2015) con el propósito de establecer medidas remediales sobre aquellos alumnos que se escapan de la media, o sobre aquellos conocimientos que a pesar de ser esperados no se reflejarían en las listas de palabras evocadas por el grupo.

\section{CONCLUSIONES}

A partir de los resultados obtenidos, se pueden extraer las siguientes conclusiones: 1) El léxico de los sujetos de la muestra aumenta a medida que aumenta su nivel académico; 2) El léxico se hace más cohesionado hacia un vocabulario especializado; y 3) EI IDLi muestra que el nivel de comunicación de los sujetos, en relación con el grupo, también aumenta.

\section{AGRADECIMIENTOS}

Los autores agradecen el financiamiento de CONICYT-PCHA/Doctorado Nacional/2014- 21141227.

\section{REFERENCIAS}

Bonorino, M. y M. Cuñarro, Las Relaciones Léxicas en los Procesos de Lectura y Escritura, Diagnóstico y Propuesta Pedagógica, Filología, 38, 251-283 (2006) 
Callealta, F. y D. Gallego, Medidas de Disponibilidad Léxica: Comparabilidad y Normalización, doi: 10.4067/S071893032016000100002, Boletín de Filología, 51(1), 39-92 (2016)

Del Valle, M., P. Salcedo y A. Ferreira, Analyzing the Availability of Lexicon in Mathematics Education Using no Traditional Technological Resources, International Journal of Supply Chain Managent, 5(2), 144-149 (2016)

Echeverría, M., M. Herrera, P. Moreno y F. Pradenas, Disponibilidad Léxica en Educación Media, RLA., Revista de Lingüística Teórica y Aplicada, 25, 55-115 (1987)

Echeverría, M., P. Urzúa e I. Figueroa, Dispogen II: Programa Computacional para el Análisis de la Disponibilidad Léxica, Concepción, Chile, Universidad de Concepción (2005)

Ferreira, A., P. Salcedo y M. del Valle, Estudio de disponibilidad léxica en el ámbito de las matemáticas, doi: 10.4067/S0071-17132014000200004, Estudios Filológicos, 54, 69-84 (2014)

Ferreira, R. y M. Echeverría, Lexical Availability of Basic and Advanced Semantic Categories in English L1 and English L2. En Jiménez Catalán (Ed.), Lexical Availability in English and Spanish as a Second Language, Springer (2014)

Garzón A. y L. Penagos, First Semester Undergraduate Students' Lexical Availability at a University in Villavicencio, Colombia, doi:10.15446/fyf.v29n2.60189, Forma y Función, 29(2), 63-84 (2016)

Gómez, MA. y L. Guerra, Disponibilidad y Fines Específicos: Análisis del Centro de Interés Prensa. III Congreso Internacional de la Asociación Europea de Lenguas para Fines Específicos (AELFE), 695-703, Universidad de Granada (2004)

Gougenheim, G., La Statistique de Vocabulaire et son Application dans l'Enseignement des Langues. Revue de l'Enseignement Supérieur, 3,137-144 (1967)

Hernández, N., C. Izura y A. Ellis, Cognitive Aspects of Lexical Availability, doi: 10.1080/09541440500339119, European Journal of Cognitive Psychology, 18(5), 730-755 (2006)

Hernández, N., El Léxico Disponible de los Estudiantes Conquenses, Salamanca, Universidad de Salamanca (2004)

Hernández, N., The Assessment of Adult Lexical Competence: An Approach from Lexical Availability and Academic Specialization in Pre-University Students, Revista de Filología de la Universidad de La Laguna, 33, 79-99 (2015)

Herranz, C., Lexical Availability in Future Teachers of Pre-Primary and Primary Education, Revista Electrónica Interuniversitaria de Formación del Profesorado, 21(1), 143-159 (2018)

Jiménez, R. y J. Dewaele, Lexical Availability of Young Spanish EFL Learners: Emotion Words Versus Non-Emotion Words, doi: 10.1080/07908318.2017.1327540, Language, Culture and Curriculum, 30(3), 283-299 (2017)

López, H., Léxico Disponible en Puerto Rico, ISBN: 84-7635-353-7, Madrid, Arco Libros (1999)

López, J. y C. Strassburger, Un modelo más para el Cálculo de Disponibilidad Léxica Individual. En H. López Morales (Ed.), La Enseñanza del Español como Lengua Materna, Río Piedras, Universidad de Puerto Rico, 91-112 (1991)

Lorán, R. y H. López, Nouveau Calcul de l'Indice de Disponibilité, Universidad de Puerto Rico (1983)

Luque, J., Aspectos Universales y Particulares de las Lenguas del Mundo, Estudios de Lingüística del Español, ISSN: 1139-8736, 21 (2004)

Medina, C., Disponibilidad Léxica Jurídica, Tesis de magister inédita, Universidad de Concepción, Chile (2009)

Mena, M., Disponibilidad Léxica Infantil en tres Niveles de Enseñanza Básica, Tesis de Magister Inédita, Universidad de Concepción, Chile (1986)

Michea, R., Mots Fréquents et Mots Disponibles, un Aspect Nouveau de la Statistique du Langage, Langues Modernes, 47, 338-344 (1953)

Navarro, Y., Terminología Especializada en el Área de Fisioterapia: Acercamiento desde la Metodología de la Disponibilidad Léxica Específica. En Interlingüística XX. Barcelona: Centre de Lingüística Teòrica, Universitat Autònoma de Barcelona, Ministerio de Ciencia e Innovación, Asociación de Jóvenes Lingüistas (2009)

Rojas, D., C. Zambrano y P. Salcedo, Metodología de Análisis de Disponibilidad Léxica en Alumnos de Pedagogía a través de la Comparación Jerárquica de Lexicones, doi: 10.4067/S0718-50062017000400002, Formación Universitaria, 10(4), 03-14 (2017)

Salcedo, P. y M., Del Valle, Disponibilidad Léxica Matemática en Estudiantes de Enseñanza Media de Concepción, Chile, Atenas, Revista Científica Pedagógica, 4 (21), 1-16 (2013)

Salcedo, P., A. Ferreira y F. Barrientos, A Bayesian Model for Lexical Availability of Chilean High School Students in Mathematics, doi:10.1007/978-3-642-38637-4_25, Lecture Notes in Computer Science, Springer Verlag, Natural and Artificial Models in Computation and Biology, 7930(1), 245-253 (2013)

Salcedo, P., M. Del Valle, A. Quintanilla y C. Zambrano, Lexical Availability of Students in Mathematics Education in Southern Chile; 9th Annual International Conference of Education, Research and Innovation, 3676-3683, Seville, Spain, 14-16 November (2016)

Šifrar Kalan, M., Lexical Availability and L2 Vocabulary Acquisition, doi:10.14706/JFLTAL152216, Foreign Language Teaching and Applied Linguistics, 192-200 (2015) 
Stoller, F., Content-Based Instruction. In Nelleke van Deusen-Scholl and Nancy H. Hornberger (Eds). Encyclopedia of Language and Education. v.4: Second and Foreign Language Education, 59-70. New York, NY, Springer (2007)

Urzúa, P., K. Sáez y M. Echeverría, Disponibilidad Léxica Matemática: Análisis Cuantitativo y Cualitativo, doi: 10.4067/S0718-48832006000200005, RLA, Revista de Lingüística Teórica y Aplicada, 44(2), 59-76 (2006)

Valencia, A. y M. Echeverría, Disponibilidad Léxica en Estudiantes de Cuarto año de Educación Media, Santiago de Chile, Universidad de Chile y Universidad de Concepción (1998)

Valencia, A., E. Ávila y otros cuatro autores, Evaluación de la Riqueza Léxica en Estudiantes de Último año de Enseñanza Media, Estudios Filológicos, (37), 61-71 (1992)

Valencia, A., Léxico del Color en Santiago de Chile, doi: 10.4067/S0718-48832010000200007, RLA, Revista de Lingüística Teórica y Aplicada, 48(2), 141-161 (2010) 
